\title{
Branch-Induced Heterogeneous Chain Motion in Precision Polyolefins
}

\author{
(Supporting Information)
}

Lucas Caire da Silva, Robert Graf§, Clifford R. Bowers and Kenneth B. Wagener*

Center for Macromolecular Science and Engineering, The George and Josephine Butler Polymer Research Laboratory, Department of Chemistry, University of Florida, Gainesville, FL 326117200

$\S$ Max Planck Institute for Polymer Research, Ackermannweg 10, 55128 Mainz, Germany

*Corresponding Author: wagener@,chem.ufl.edu 


\section{Instrumentation}

All NMR $\left({ }^{1} \mathrm{H}, 300 \mathrm{MHz}\right.$ and $\left.{ }^{13} \mathrm{C}, 75 \mathrm{MHz}\right)$ spectra were recorded in $\mathrm{CDCl}_{3}$ at room temperature and referenced to the corresponding residual signals $\left(\mathrm{CDCl}_{3}:{ }^{1} \mathrm{H}\right.$ at $7.24 \mathrm{ppm},{ }^{13} \mathrm{C}$ at $77.23 \mathrm{ppm}$ ). High-resolution mass spectrometry was carried out in a Thermo Scientific Trace DSQ GC-MS spectrometer. Elemental analysis was performed by combustion using automatic analyzer. Gel permeation chromatography (GPC) was performed using an Agilent 1100 Series HPLC System $\left(\mathrm{THF}, 30^{\circ} \mathrm{C}\right)$ equipped with internal differential refractive index detector. Retention times were calibrated versus polystyrene standards. Differential Scanning Calorimetry (DSC) analyses were performed on a TA Instruments Q1000 calorimeter. All the analyses were obtained under He purge at a rate of $10^{\circ} \mathrm{C} \cdot \mathrm{min}^{-1}$ with samples prepared in hermetically sealed pans. Samples were analyzed from $-80^{\circ} \mathrm{C}$ to $300^{\circ} \mathrm{C}$ with 5 minutes isothermal steps at the temperature extremes for each cycle. The data used in this work were obtained from the last of the three heating cycles performed. Calibration was performed with indium as the standard for both the heats of fusion and peak temperature transitions. Thermogravimetric analysis (TGA) was performed on a TA Instruments Q5000 analyzer. All samples were analyzed with a temperature range from room temperature to $650^{\circ} \mathrm{C}$ in nitrogen at $20^{\circ} \mathrm{C} /$ minutes with a purge rate of $20 \mathrm{~mL} / \mathrm{min}$. 


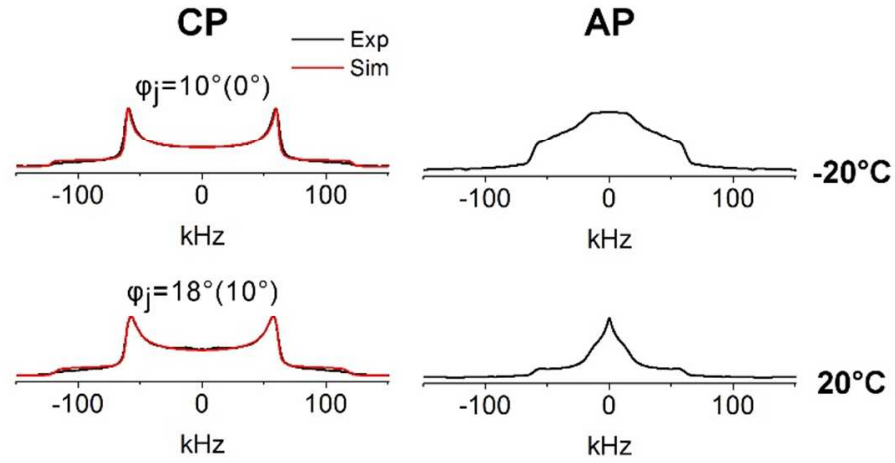

Figure S1. Experimental and simulated CP/AP lineshapes of PE21- $\mathrm{D}_{2}$ at $-20^{\circ} \mathrm{C}$ and $20^{\circ} \mathrm{C}$.

Simulation assumed the fast-motion regime at $20^{\circ} \mathrm{C}$ and $500 \mathrm{kHz}$ motion-rate at $-20^{\circ} \mathrm{C}$.

The $500 \mathrm{kHz}$ motion-rate used in the simulation of PE21- $\mathrm{D}_{2}$ at $-20^{\circ} \mathrm{C}$ (Figure S1) allows certain variation. The figure below shows the $\%$ error obtained for different motion-rates.

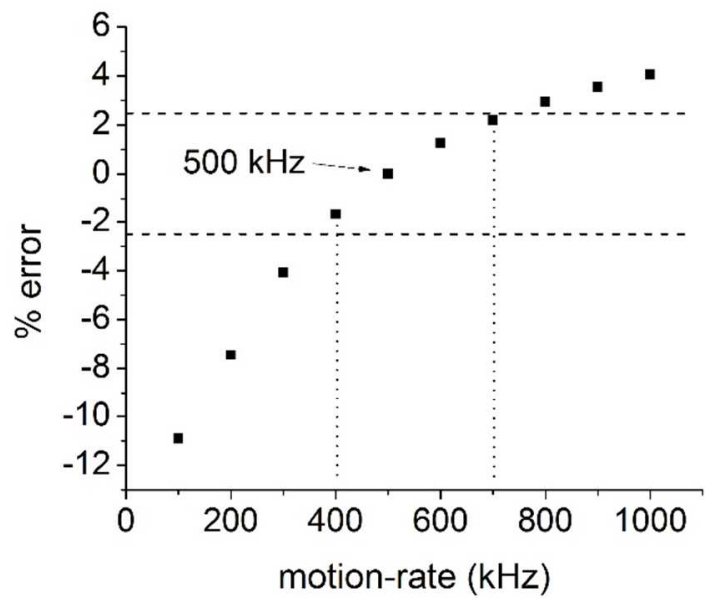

Figure S2. Signal intensity \% error obtained when different motion-rates are considered. Values are relative to the signal intensity at the center of the pattern. Dashed lines define a $\pm 2.5 \%$ error limit. 


\section{Materials}

Chemical reagents used in this work were purchased from Sigma-Aldrich Co. and used as received, unless specified otherwise. Hexamethylphosphoramide, tert-butanol and propionitrile were distilled over $\mathrm{CaH}_{2}$. Dry solvents (tetrahydrofuran, dichloromethane, diethyl ether and toluene) were obtained from the Butler Laboratories' Solvent Purification System. Schrock's catalyst, 2,6-Diisopropylphenylimidoneophylidene molybdenum(VI) bis(hexafluoro- $t$-butoxide, was purchased from Strem Chemicals, Inc.

\section{1 - Synthesis of deuterated spacer bromides}

\section{Oct-7-en-1,1-d $d_{2}$-1-ol (1a)}<smiles>[2H]C([2H])(O)CCCCCC=C</smiles>

oct-7-en-1,1-d $d_{2}-1-$ ol Chemical Formula: $\mathrm{C}_{8} \mathrm{H}_{14} \mathrm{D}_{2} \mathrm{O}$

Molecular Weight: 130.23
A solution of oct-7-enoic acid (5g, $35.2 \mathrm{mmol}, 1$ equivalent) in THF (30 mL) was slowly added into a flame-dried round-bottom flask containing lithium aluminum deuteride (LAD) dissolved in THF ( 1 mol.L $\mathrm{L}^{-1}, 49.2 \mathrm{~mL}, 1.4$ equivalents). The temperature of the LAD solution was kept at $0^{\circ} \mathrm{C}$ during the addition of the acid. The reaction was allowed to proceed at room temperature under argon atmosphere and followed by TLC (thin layer chromatography). After one hour, the reaction mixture was quenched with $2 \mathrm{~mL}$ of deionized water (DIW), $2 \mathrm{~mL}$ of $\mathrm{NaOH}$ solution $(15 \% \mathrm{w} / \mathrm{v})$ and $6 \mathrm{~mL}$ of DIW, in that order. The mixture was filtered and concentrated to an oil which was subsequently dissolved in ether. The solution was dried over $\mathrm{MgSO}_{4}$ for 45 minutes and filtered prior to evaporation. A white solid was obtained (4.35 g, 95\%). No further purification was necessary. ${ }^{1} \mathrm{H}$ NMR (300 MHz, CHLOROFORM- $d$ ) $\delta$ ppm $1.17-1.45$ (br, 6H) 1.54 (t, 2H, $J=5.8 \mathrm{~Hz}) 2.04(\mathrm{q}, 2 \mathrm{H}, J=6.90 \mathrm{~Hz}) 4.84-5.08(\mathrm{~m}, 2 \mathrm{H}) 5.69-5.94$ (ddt, 1H, $J=16.90,10.10,6.60$ Hz). ${ }^{13} \mathrm{C}$ NMR (75 MHz, CHLOROFORM-d) $\delta$ ppm 25.6, 28.92, 29.11, 29.41, 29.54, 32.58, 33.80, 114.06, 139.17. CG/CI-MS: [M+1] calculated for $\mathrm{C}_{8} \mathrm{H}_{14} \mathrm{D}_{2} \mathrm{O}, \mathrm{m} / \mathrm{z} 131.1405$; found $\mathrm{m} / \mathrm{z}$ 131.1405. Elemental Analysis: calculated for $\mathrm{C}_{8} \mathrm{H}_{14} \mathrm{D}_{2} \mathrm{O}$ : C, 73.76; $(\mathrm{H}+\mathrm{D})$, 12.41. Found: $\mathrm{C}$, 73.94; (H+D), 12.40 . 
8-bromooct-1-ene-8,8- $d_{2}(1 b)$

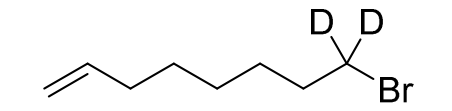

8-bromooct-1-ene-8,8- $d_{2}$

Chemical Formula: $\mathrm{C}_{8} \mathrm{H}_{13} \mathrm{D}_{2} \mathrm{Br}$

Molecular Weight: 193.12

A solution of 1a $(4.36 \mathrm{~g}, 33.48 \mathrm{mmol})$ and $\mathrm{CBr}_{4}(12.10 \mathrm{~g}, 36.49$ $\mathrm{mmol})$ in dichloromethane $(15 \mathrm{~mL})$ was prepared in a $50 \mathrm{~mL}$ round bottom flask equipped with a magnetic stirrer. This solution was cooled and maintained at $0^{\circ} \mathrm{C}$ in an ice bath. Triphenylphosphine (9.66 g, $36.83 \mathrm{mmol})$ was added to the cold solution in small portions through a powder funnel. After addition

of the triphenylphosphine, the pale brown reaction mixture was stirred for an additional 2 hours at room temperature. The crude was then quickly poured into stirring hexanes. The precipitated solid was filtered and the liquid obtained was concentrated and purified by column chromatography and distilled under reduced pressure. Compound $\mathbf{1 b}$ was obtained as a clear liquid (3.51 g, 54\%). ${ }^{1} \mathrm{H}$ NMR (300 MHz, CHLOROFORM- $d$ ) $\delta$ ppm $1.22-1.53$ (br, 6H) 1.85 $(\mathrm{t}, 2 \mathrm{H}, J=6.8 \mathrm{~Hz}) 1.96-2.18(\mathrm{q}, 2 \mathrm{H}, J=6.9 \mathrm{~Hz}) 4.83-5.15(\mathrm{~m}, 2 \mathrm{H}) 5.65-6.01$ (ddt, $1 \mathrm{H}, J_{I}=16.9$, $\left.J_{2}=10.1, J_{3}=6.7 \mathrm{~Hz}\right) .{ }^{13} \mathrm{C}$ NMR $(75 \mathrm{MHz}$, CHLOROFORM- $d$ ) $\delta \mathrm{ppm} 27.95,28.22,28.67,32.52$, 33.64, 114.35, 138.83. CG/CI-MS: $[\mathrm{M}]^{+}$calcd for $\mathrm{C}_{8} \mathrm{H}_{12} \mathrm{D}_{2} \mathrm{Br}$, 192.0483; found 192.0483. Elemental Analysis: calcd for $\mathrm{C}_{8} \mathrm{H}_{13} \mathrm{D}_{2} \mathrm{Br}$ : C, 49.75; (H+D), 7.84. Found: C, 49.85; (H+D), 7.68.

\section{Unde-10-en-1,1- $d_{2}$-1-ol (1c)}

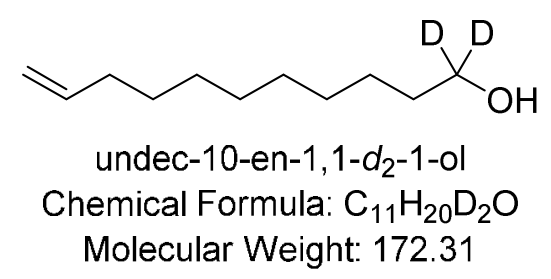

Zinc undecylenate (18.89 g, $43.8 \mathrm{mmol})$ was slowly added through a powder funnel into a LAD solution in THF $(1 \mathrm{M}, 50$ $\mathrm{mL})$ at $0^{\circ} \mathrm{C}$. The mixture was allowed to warm to room temperature and kept stirring under argon for one hour. The reaction was quenched with $2 \mathrm{~mL}$ of deionized water, $2 \mathrm{~mL}$ of $\mathrm{NaOH} 15 \%(\mathrm{w} / \mathrm{v})$ and $6 \mathrm{~mL}$ deionized water in that order with 10 minutes intervals between each addition. The crude mixture was filtered and concentrated. The oil obtained was dissolved in ether and dried over $\mathrm{MgSO}_{4}$ for 45 minutes. The product was purified by column chromatography. A white solid was obtained $(12.38 \mathrm{~g}, 82.1 \%)$. ${ }^{1} \mathrm{H}$ NMR (300 MHz, CHLOROFORM- $d$ ) $\delta$ ppm 1.30 (br., 12H) 1.55 (t, 2H, $J=6.1 \mathrm{~Hz}$ ) 2.04 (q, 2H, $J=6.7$ $\mathrm{Hz}) 4.85-5.08(\mathrm{~m}, 2 \mathrm{H}) 5.70-5.93\left(\mathrm{ddt}, 1 \mathrm{H}, J_{I}=17.1, J_{2}=10.4, J_{3}=6.7 \mathrm{~Hz}\right) .{ }^{13} \mathrm{C}$ NMR $(75 \mathrm{MHz}$, CHLOROFORM- $d$ ) $\delta$ ppm 25.67, 28.90, 29.09, 29.40, 29.53, 32.54, 33.78, 114.05 (vinyl $\mathrm{CH}_{2}$ ), 139.14 (vinyl $\mathrm{CH}$ ). CG/CI-MS: $[\mathrm{M}+\mathrm{H}]^{+}$calcd for $\mathrm{C}_{11} \mathrm{H}_{21} \mathrm{D}_{2} \mathrm{O}$, 173.1874; found 173.1882 . Elemental Analysis: calcd for $\mathrm{C}_{11} \mathrm{H}_{20} \mathrm{D}_{2} \mathrm{O}$ : C, 76.68; $(\mathrm{H}+\mathrm{D}), 12.89$. Found: $\mathrm{C}, 76.57$; $(\mathrm{H}+\mathrm{D})$, 12.76

\section{1-bromoundec-1-ene-11,11- $d_{2}$ (1d)}

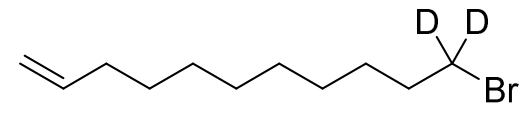

11-bromoundec-1-ene-11,11- $d_{2}$ Chemical Formula: $\mathrm{C}_{11} \mathrm{H}_{19} \mathrm{D}_{2} \mathrm{Br}$

Molecular Weight: 235.21
The synthesis followed the same procedure used for $\mathbf{1 b}$. Compound 1d was prepared from a solution of $1 \mathrm{c}(6.00 \mathrm{~g}, 34.82$ $\mathrm{mmol})$ and $\mathrm{CBr}_{4}(12.59 \mathrm{~g}, 37.59 \mathrm{mmol})$ in dichloromethane (15 
$\mathrm{mL}$ ). Compound 1d was obtained as a clear liquid (6.15 g, 75\%). ${ }^{1} \mathrm{H}$ NMR (300 MHz, CHLOROFORM- $d$ ) $\delta$ ppm $1.19-1.51(\mathrm{~m}, 12 \mathrm{H}) 1.77-1.91(\mathrm{t}, 2 \mathrm{H}, J=7.4 \mathrm{~Hz}) 2.05(\mathrm{q}, 2 \mathrm{H}, J=6.7$ $\mathrm{Hz}) 4.86-5.08(\mathrm{~m}, 2 \mathrm{H}) 5.73-5.93\left(\mathrm{ddt}, 1 \mathrm{H}, J_{1}=16.9, J_{2}=10.4, J_{3}=6.7 \mathrm{~Hz}\right) .{ }^{13} \mathrm{C}$ NMR $(75 \mathrm{MHz}$, CHLOROFORM- $d$ ) $\delta$ ppm 28.11, 28.75, 28.90, 29.08, 29.37, 32.59, 33.79, 114.11, 139.13. CG/CI-MS: $[\mathrm{M}+\mathrm{H}]^{+}$calcd for $\mathrm{C}_{11} \mathrm{H}_{19} \mathrm{D}_{2} \mathrm{Br}$, 234.0952; found 234.0954. Elemental Analysis: calcd for $\mathrm{C}_{11} \mathrm{H}_{19} \mathrm{D}_{2} \mathrm{Br}$ : C, 56.16; (H+D), 9.02; Br, 33.97. Found: C, 56.21; (H+D), 8.81; $\mathrm{Br}$, 33.80 .

\section{$\underline{2}$ - Synthesis of non-deuterated spacer bromides}

\section{8-bromooct-1-ene (2a)}<smiles>C=CCCCCCCBr</smiles>

8-bromooct-1-ene Chemical Formula: $\mathrm{C}_{8} \mathrm{H}_{15} \mathrm{Br}$ Molecular Weight: 191.11

A $1 \mathrm{M}$ solution of 1,8-dibromooctane (100 g, $0.367 \mathrm{~mol})$ using 1:1 THF:Toluene as the solvent was added to a round bottom flask and cooled to $0^{\circ} \mathrm{C}$. Potassium tert-butoxide $(61.88,0.551 \mathrm{~mol})$ was added in small portions over 30 minutes. After the addition, the reaction was quenched with $1 \mathrm{M} \mathrm{HCl}$. The mixture was extracted with ether and washed with saturated $\mathrm{Na}_{2} \mathrm{CO}_{3}$. The organic layer was then dried over $\mathrm{MgSO}_{4}$, concentrated and distilled. Compound 2a was obtained as a clear liquid (50 g, 71\%). ${ }^{1} \mathrm{H} \mathrm{NMR}$ (300 MHz, CHLOROFORM- $d$ ) $\delta$ ppm 1.21 - 1.53 (m, 6 H) 1.87 (quin, 2 H) 2.06 (q, 2 H) 3.42 (t, $2 \mathrm{H}) 4.88$ - 5.11 (m, $2 \mathrm{H}) 5.81$ (ddt, $1 \mathrm{H}) .{ }^{13} \mathrm{C}$ NMR (75 MHz, CHLOROFORM- $d$ ) $\delta$ ppm 28.23, 28.44, 28.90, 32.98, 33.87, 34.20, 114.57, 139.05. CG/CI-MS: [M] ${ }^{+}$calcd for $\mathrm{C}_{8} \mathrm{H}_{15} \mathrm{Br}$, 190.0357; found 190.0351. Elemental Analysis: calcd for $\mathrm{C}_{8} \mathrm{H}_{15} \mathrm{Br}$ : C, 50.28; H, 7.91; $\mathrm{Br}, 41.81$. Found: C, 50.38; H, 7.97; Br, 41.57.

\section{1-bromoundec-1-ene (2b)}

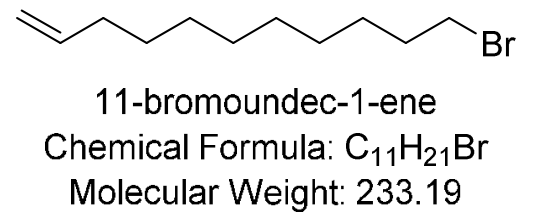

The synthesis followed the same procedure used for $\mathbf{1 b}$. Compound $\mathbf{2 b}$ was prepared from a solution of 10-undecen-1-ol $(100 \mathrm{~g}, 0.587 \mathrm{~mol})$ and $\mathrm{CBr}_{4}(212.8 \mathrm{~g}, 0.639 \mathrm{~mol})$ in dichloromethane $(250 \mathrm{~mL})$. Compound $\mathbf{2 b}$ was obtained as a clear liquid $(50 \quad \mathrm{~g}, \quad 36 \%) .{ }^{1} \mathrm{H} \quad \mathrm{NMR} \quad(300 \quad \mathrm{MHz}$, CHLOROFORM-d) $\delta$ ppm $1.18-1.52$ (br, 12H) 1.86 (quint, 2H, $J=7.1 \mathrm{~Hz}$ ) 2.05 (q, 2H, $J=6.86$ $\mathrm{Hz}) 3.42(\mathrm{t}, 2 \mathrm{H}, J=6.86 \mathrm{~Hz}) 4.86-5.09(\mathrm{~m}, 2 \mathrm{H}) 5.74-5.92\left(\mathrm{ddt}, 1 \mathrm{H}, J_{I}=16.94, J_{2}=10.2, J_{3}=6.7\right.$ Hz). ${ }^{13} \mathrm{C}$ NMR (75 MHz, CHLOROFORM-d) $\delta$ ppm 28.17, 28.75, 28.91, 29.08, 29.37, 32.83, 33.79, 34.06, 114.11, 139.14. CG/CI-MS: [M] ${ }^{+}$calcd for $\mathrm{C}_{11} \mathrm{H}_{21} \mathrm{Br}$, 232.0827; found 232.0819. Elemental Analysis: calcd for $\mathrm{C}_{11} \mathrm{H}_{21} \mathrm{Br}$ : C, 56.66; H, 9.08; Br, 34.27. Found: C, 56.92; H, 9.21; $\mathrm{Br}, 34.40$. 


\section{$\underline{3}$ - Synthesis of pre-monomers}

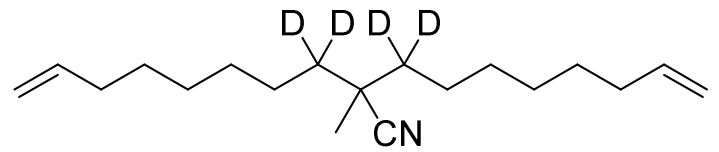

2-methyl-2-(oct-7-en-1-yl-1,1- $d_{2}$ )dec-9-enenitrile-3,3- $d_{2}$ Chemical Formula: $\mathrm{C}_{19} \mathrm{H}_{29} \mathrm{D}_{4} \mathrm{~N}$

Molecular Weight: 279.50

2-methyl-2-(oct-7-en-1-yl-1,1-d $\left.d_{2}\right)$ dec-9enenitrile-3,3- $d_{2}$ (3a)

A solution of propionitrile $(0.41 \mathrm{~g}, 7.44$ mmol) in THF $(10 \mathrm{~mL})$ was prepared in a flame-dried three-necked round-bottomed flask equipped with a stir bar and argon inlet adaptor. The solution was cooled to $78^{\circ} \mathrm{C}$ and $5 \mathrm{~mL}$ of lithium diisopropylamine (LDA) solution in THF $\left(2\right.$ mol. $\left.\mathrm{L}^{-1}\right)$ was added. The temperature of the mixture was increased and maintained at $0^{\circ} \mathrm{C}$ for 45 minutes. After that, the reaction was cooled to $-78^{\circ} \mathrm{C}$ and $\mathbf{1 b}(1.76 \mathrm{~g}$, $9.08 \mathrm{mmol}$ ) was added. The temperature was increased to $0^{\circ} \mathrm{C}$ and maintained at this temperature for another 45 minutes. A second $5 \mathrm{~mL}$ portion of the LDA solution was added at $-78^{\circ} \mathrm{C}$. The temperature was increased and maintained at $0^{\circ} \mathrm{C}$ for 45 minutes. The temperature was returned to $-78^{\circ} \mathrm{C}$ and $\mathbf{1 b}(1.76 \mathrm{~g}, 9.08 \mathrm{mmol})$ was added. The reaction mixture was warmed to $0^{\circ} \mathrm{C}$ for 30 minutes and proceeded overnight at room temperature. The reaction was quenched with deionized water, extracted with ether and dried over $\mathrm{MgSO}_{4}$. The crude was concentrated and purified by flash-column chromatography. Compound 3a was obtained as pale yellow liquid (6.26 g, 81.3\%). ${ }^{1} \mathrm{H}$ NMR (300 MHz, CHLOROFORM- $d$ ) $\delta$ ppm 1.05 - 1.55 (br, $\left.19 \mathrm{H}\right) 2.05$ (q, $4 \mathrm{H}, J=6.1 \mathrm{~Hz}) 4.78-5.11(\mathrm{~m}, 4 \mathrm{H}) 5.63-6.08\left(\mathrm{ddt}, 2 \mathrm{H}, J_{l}=16.9, J_{2}=10.1, J_{3}=6.6 \mathrm{~Hz}\right) .{ }^{13} \mathrm{C} \mathrm{NMR}$ $(75 \mathrm{MHz}$, CHLOROFORM- $d$ ) $\delta$ ppm 24.10, 24.80, 29.02, 29.12, 29.73, 33.94, 36.60, 114.49, 124.91, 139.16. CG/CI-MS: $[\mathrm{M}+\mathrm{H}]^{+}$calcd for $\mathrm{C}_{19} \mathrm{H}_{30} \mathrm{D}_{4} \mathrm{~N}, 280.2942$; found 280.2949. Elemental Analysis: calcd for $\mathrm{C}_{19} \mathrm{H}_{29} \mathrm{D}_{4} \mathrm{~N}$ : C, 81.65; (H+D), 11.92; N, 5.01. Found: C, 81.62; $(\mathrm{H}+\mathrm{D}), 12.00$; N, 4.86 .

\section{2-methyl-2-(undec-10-en-1-yl-1,1- $\left.d_{2}\right)$ tridec-12-enenitrile-3,3- $d_{2}(3 \mathrm{~b})$}

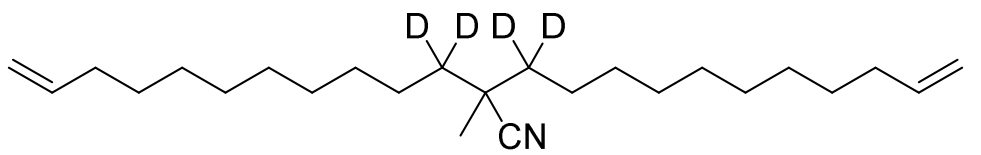

2-methyl-2-(undec-10-en-1-yl-1,1- $d_{2}$ )tridec-12-enenitrile-3,3- $d_{2}$ Chemical Formula: $\mathrm{C}_{25} \mathrm{H}_{41} \mathrm{D}_{4} \mathrm{~N}$

Molecular Weight: 363.67

The synthesis followed the same procedure used for 3a. Compound 3b was prepared from a solution of propionitrile $(0.151 \mathrm{~g}, 2.75 \mathrm{mmol})$ in THF $(2.75 \mathrm{~mL}), 2$ mol.L $\mathrm{L}^{-1}$ LDA solution in THF $(3.36 \mathrm{~mL}, 6.71$ mmol) and 1d (1.58 g, $6.71 \mathrm{mmol})$. Compound 3b was obtained as a pale yellow liquid $(800 \mathrm{mg} 80 \%) .{ }^{1} \mathrm{H}$ NMR $(300 \mathrm{MHz}$, CHLOROFORM- $d$ ) $\delta$ ppm $1.13-1.50$ (br, 31H) 2.05 (q, 4H, J=6.9 Hz) 4.87 - 5.07 (m, 4H) 5.72 - $5.92\left(\mathrm{ddt}, 2 \mathrm{H}, J_{l}=16.9, J_{2}=10.2, J_{3}=6.7 \mathrm{~Hz}\right) .{ }^{13} \mathrm{C}$ NMR $(75 \mathrm{MHz}, \mathrm{CHLOROFORM}-d) \delta \mathrm{ppm}$ 
24.10, 24.83, 29.15, 29.33, 29.64, 29.66, 29.70, 29.88, 34.03, 36.61, 114.31, 124.97, 139.38 . CG/CI-MS: $[\mathrm{M}+\mathrm{H}]^{+}$calcd for $\mathrm{C}_{25} \mathrm{H}_{42} \mathrm{D}_{4} \mathrm{~N}, 364.3881$; found 364.3893. Elemental Analysis: calcd for $\mathrm{C}_{25} \mathrm{H}_{41} \mathrm{D}_{4} \mathrm{~N}$ : C, 82.57; (H+D), 12.49; N, 3.85. Found: C, 82.77; (H+D), 12.63; N, 3.87 .

2-methyl-2-(oct-7-en-1-yl)dec-9-enenitrile (3c)

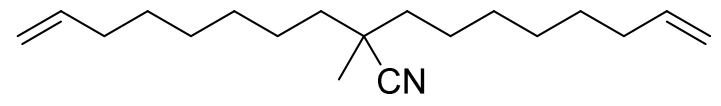

2-methyl-2-(oct-7-en-1-yl)dec-9-enenitrile Chemical Formula: $\mathrm{C}_{19} \mathrm{H}_{33} \mathrm{~N}$ Molecular Weight: 275.48
The synthesis followed the same procedure used for 3a. Compound 3c was prepared from a solution of propionitrile $(1.18 \mathrm{~g}, 21.42 \mathrm{mmol})$ in THF $(20 \mathrm{~mL})$, 2 mol. $\mathrm{L}^{-1}$ LDA solution in THF $(26.16 \mathrm{~mL}, 52.32$ mmol) and 2a (10 g, $52.32 \mathrm{mmol})$. Compound 3c was obtained as a pale yellow liquid (5.48 $\mathrm{g}$, 93\%). ${ }^{1} \mathrm{H}$ NMR (300 MHz, CHLOROFORM-d) $\delta$ ppm 1.19 - 1.54 (br, 23H) $1.97-2.13$ (q, 4H, $J=6.6 \mathrm{~Hz}) 4.87-5.07(\mathrm{~m}, 4 \mathrm{H}) 5.82\left(\mathrm{ddt}, 2 \mathrm{H}, J_{l}=17, J_{2}=10.2, J_{3}=6.7 \mathrm{~Hz}\right) .{ }^{13} \mathrm{C} \mathrm{NMR}(75 \mathrm{MHz}$, CHLOROFORM- $d$ ) $\delta$ ppm 23.98, 24.78, 28.79, 28.89, 29.54, 33.72, 36.70, 39.42, 114.27, 124.66, 138.93. CG/CI-MS: $[\mathrm{M}+\mathrm{H}]^{+}$calcd for $\mathrm{C}_{19} \mathrm{H}_{34} \mathrm{~N}, 276.2691$; found 276.2705. Elemental Analysis: calcd for $\mathrm{C}_{19} \mathrm{H}_{33} \mathrm{~N}$ : C, 82.84; H, 12.07. Found: C, 82.99; H, 12.13.

\section{2-methyl-2-(undec-10-en-1-yl)tridec-12-enenitrile (3d)}

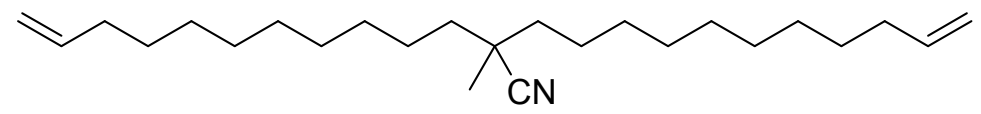

2-methyl-2-(undec-10-en-1-yl)tridec-12-enenitrile

Chemical Formula: $\mathrm{C}_{25} \mathrm{H}_{45} \mathrm{~N}$

Molecular Weight: 359.64
The synthesis followed the same procedure used for 3a. Compound 3d was prepared from a solution of propionitrile $(0.968 \mathrm{~g}, 17.57 \mathrm{mmol})$ in THF $(18 \mathrm{~mL}), 2 \mathrm{~mol} . \mathrm{L}^{-1}$ LDA solution in THF $(35.50 \mathrm{~mL}, 42.88$

mmol) and $\mathbf{2 b}(10 \mathrm{~g}, 42.88 \mathrm{mmol})$. Compound 3d was obtained as a pale yellow liquid (99\%). ${ }^{1} \mathrm{H}$ NMR (300 MHz, CHLOROFORM- $d$ ) $\delta$ ppm 1.10 - 1.52 (br, 35H) 2.05 (q, 4H, J=6.8 Hz) $4.87-5.07(\mathrm{~m}, 4 \mathrm{H}) 5.72-5.92\left(\mathrm{ddt}, 2 \mathrm{H}, J_{l}=17.1, J_{2}=10.4, J_{3}=6.7 \mathrm{~Hz}\right) .{ }^{13} \mathrm{C} \mathrm{NMR}(75 \mathrm{MHz}$, CHLOROFORM-d) $\delta$ ppm 23.98, 24.80, 28.91, 29.09, 29.40, 29.43, 29.47, 29.69, 33.79, 36.69, 39.43, 114.08, 124.69, 139.13. CG/CI-MS: $[\mathrm{M}+\mathrm{H}]^{+}$calcd for $\mathrm{C}_{25} \mathrm{H}_{46} \mathrm{~N}$, 360.3630; found 360.3624. Elemental Analysis: calcd for $\mathrm{C}_{25} \mathrm{H}_{45} \mathrm{~N}$ : C, 83.49; H, 12.61; N, 3.89. Found: C, 83.36; H, 12.65; N, 3.96.

\section{$\underline{4-\text { Synthesis of monomers }}$}

\section{9-methylheptadeca-1,16-diene-8,8,10,10- $d_{4}(4 a)$}




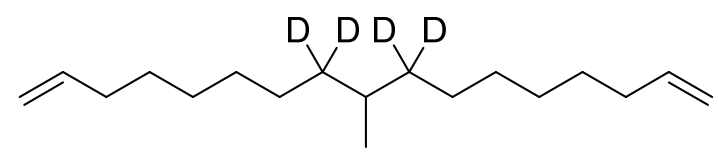

9-methylheptadeca-1,16-diene-8,8,10,10- $d_{4}$ Chemical Formula: $\mathrm{C}_{18} \mathrm{H}_{30} \mathrm{D}_{4}$ Molecular Weight: 254.49

Hexamethylphosphoramide (HMPA) (5.53 g, 30.84 mmol), potassium metal chunks (1.68 g, 42.93 $\mathrm{mmol})$ and ether $(40 \mathrm{~mL})$ were added to a flamedried three-necked round-bottomed flask equipped with stir bar, argon inlet adaptor and addition funnel. A solution of 3a (1.69 g, $6.05 \mathrm{mmol})$ and tertbutanol $(1.17 \mathrm{~g}, 15.72 \mathrm{mmol})$ in ether $(30 \mathrm{~mL})$ was transferred to the addition funnel and added dropwise at $0^{\circ} \mathrm{C}$ under argon atmosphere. After the addition, the reaction was allowed to proceed for 4 hours at $0^{\circ} \mathrm{C}$ and overnight at room temperature. The remaining pieces of potassium were removed and the reaction was quenched with deionized water. The organic layer was extracted with ether, dried over $\mathrm{MgSO}_{4}$, concentrated and purified by flash-column chromatography (hexanes). Compound 4a was obtained as a clear liquid (1.46 g, 95\%). ${ }^{1} \mathrm{H}$ NMR (300 MHz, CHLOROFORM- $d$ ) $\delta$ ppm $0.84(\mathrm{~d}, 3 \mathrm{H}, J=6.6 \mathrm{~Hz}) 1.14-1.48$ (br, 17H) 2.05 (q, 4H, $J=7.0 \mathrm{~Hz}$ ) $4.86-5.11(\mathrm{~m}, 4 \mathrm{H}) 5.83\left(\mathrm{ddt}, 2 \mathrm{H}, J_{l}=17, J_{2}=10.2, J_{3}=6.7 \mathrm{~Hz}\right) .{ }^{13} \mathrm{C}$ NMR $(75 \mathrm{MHz}$, CHLOROFORM- $d$ ) $\delta$ ppm 19.63, 26.84, 28.99, 29.23, 29.83, 32.38, 33.85, 114.05, 139.23. CG/CI-MS: [M] $]^{+}$calcd for $\mathrm{C}_{18} \mathrm{H}_{30} \mathrm{D}_{4}, 254.2912$; found 254.2913. Elemental Analysis: calcd for $\mathrm{C}_{18} \mathrm{H}_{30} \mathrm{D}_{4}$ : C, 84.92; (H+D), 13.49. Found: $\mathrm{C}, 85.13$; $(\mathrm{H}+\mathrm{D}), 13.73$

\section{2-methyltricosa-1,22-diene-11,11,13,13- $d_{4}(4 \mathrm{~b})$}

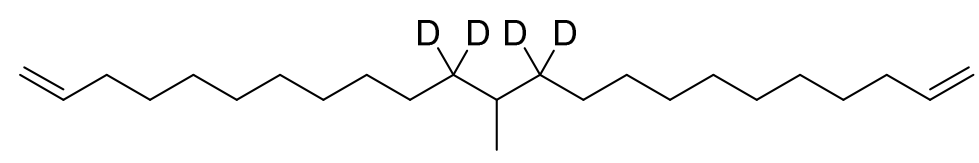

12-methyltricosa-1,22-diene-11,11,13,13- $d_{4}$ Chemical Formula: $\mathrm{C}_{24} \mathrm{H}_{42} \mathrm{D}_{4}$ Molecular Weight: 338.66
The synthesis followed the same procedure used for $\mathbf{4 a}$. Compound $\mathbf{4 b}$ was prepared by addition of a solution of $\mathbf{3 b}(2.60 \mathrm{~g}, 7.15 \mathrm{mmol})$, tert-butanol (1.38 g, $18.61 \mathrm{mmol})$ in ether $(30 \mathrm{~mL})$ into a mixture of

HMPA $(6.53 \mathrm{~g}, 36.44 \mathrm{mmol})$ and $\mathrm{K}(2.0 \mathrm{~g}, 51.16 \mathrm{mmol})$ in ether $(45 \mathrm{~mL})$. Compound $\mathbf{4 b}$ was obtained as a clear liquid $(2.18 \mathrm{~g}, 90 \%) .{ }^{1} \mathrm{H}$ NMR $(300 \mathrm{MHz}, \mathrm{CHLOROFORM}-d) \delta \mathrm{ppm} 0.84(\mathrm{~d}$, $3 \mathrm{H}, J=6.6 \mathrm{~Hz}) 1.14-1.48(\mathrm{~m}, 29 \mathrm{H}) 2.05(\mathrm{q}, 4 \mathrm{H}, J=6.8 \mathrm{~Hz}) 4.86-5.09(\mathrm{~m}, 4 \mathrm{H}) 5.70$ - 5.93 (ddt, $\left.2 \mathrm{H}, J_{I}=17.1, J_{2}=10.2, J_{3}=6.7 \mathrm{~Hz}\right) .{ }^{13} \mathrm{C}$ NMR $(75 \mathrm{MHz}$, CHLOROFORM- $d$ ) $\delta$ ppm 19.86, 27.11, 29.19, 29.40, 29.76, 29.87, 29.96, 30.21, 32.61, 34.06, 114.26, 139.45. CG/CI-MS: [M] ${ }^{+}$calcd for $\mathrm{C}_{24} \mathrm{H}_{42} \mathrm{D}_{4}, 338.3850$; found 338.3850. Elemental Analysis: calcd for $\mathrm{C}_{24} \mathrm{H}_{42} \mathrm{D}_{4}$ : C, 85.09; $(\mathrm{H}+\mathrm{D}), 13.72$. Found: C, 85.24; (H+D), 14.01 .

\section{9-methylheptadeca-1,16-diene (4c)}

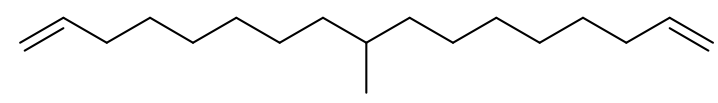

9-methylheptadeca-1,16-diene Chemical Formula: $\mathrm{C}_{18} \mathrm{H}_{34}$ Molecular Weight: 250.47
The synthesis followed the same procedure used for 4a. Compound $4 \mathbf{c}$ was prepared by addition of a solution of 3c (5.48 g, $19.89 \mathrm{mmol})$, tert-butanol (3.83 g, $51.67 \mathrm{mmol})$ in ether $(85 \mathrm{~mL})$ into a mixture of HMPA (18.18 g, $101.5 \mathrm{mmol})$ and $\mathrm{K}(5.5 \mathrm{~g}, 140.7$ $\mathrm{mmol})$ in ether $(120 \mathrm{~mL})$. Compound $4 \mathbf{c}$ was obtained as a clear liquid $(3.87 \mathrm{~g}, 78 \%) .{ }^{1} \mathrm{H}$ NMR 
(300 MHz, CHLOROFORM- $d$ ) $\delta$ ppm 0.85 (d, 3H, $J=6.3 \mathrm{~Hz}) 0.98-1.47$ (br, 21H) 2.05 (q, 4H, $J=6.8 \mathrm{~Hz}) 4.87-5.08(\mathrm{~m}, 4 \mathrm{H}) 5.83\left(\mathrm{ddt}, 2 \mathrm{H}, J_{I}=17.0, J_{2}=10.2, J_{3}=6.6 \mathrm{~Hz}\right) .{ }^{13} \mathrm{C}$ NMR $(75 \mathrm{MHz}$, CHLOROFORM- $d$ ) $\delta$ ppm 19.93, 27.25, 29.19, 29.43, 30.08, 32.96, 34.06, 37.28, 114.26, 138.44. CG/EI-MS: [M] $]^{+}$calcd for $\mathrm{C}_{18} \mathrm{H}_{34}, 250.2660$; found 250.2648. Elemental Analysis: calcd for $\mathrm{C}_{18} \mathrm{H}_{34}: \mathrm{C}, 86.32 ; \mathrm{H}, 13.68$. Found: $\mathrm{C}, 86.29 ; \mathrm{H}, 13.49$.

\section{2-methyltricosa-1,22-diene (4d)}

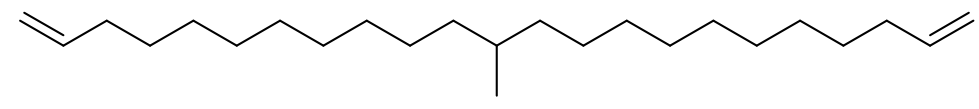

12-methyltricosa-1,22-diene

Chemical Formula: $\mathrm{C}_{24} \mathrm{H}_{46}$

Molecular Weight: 334.63
The synthesis followed the same procedure used for $\mathbf{4 a}$. Compound $\mathbf{4 d}$ was prepared by addition of a solution of $\mathbf{3 d}(4.38 \mathrm{~g}, 12.18 \mathrm{mmol})$, tert-butanol (2.35 g, $31.71 \mathrm{mmol})$ in ether $(55 \mathrm{~mL})$ into a mixture of

HMPA (11.13 g, $62.11 \mathrm{mmol})$ and $\mathrm{K}(3.38 \mathrm{~g}, 86.47 \mathrm{mmol})$ in ether $(75 \mathrm{~mL})$. Compound 4d was obtained as a clear $(3.0 \mathrm{~g}, 74 \%) .{ }^{1} \mathrm{H}$ NMR $(300 \mathrm{MHz}$, CHLOROFORM- $d$ ) $\delta$ ppm $0.85(\mathrm{~d}, 3 \mathrm{H}$, $J=6.4 \mathrm{~Hz}) 1.00-1.47$ (br, 33H) 2.05 (q, 4H, $J=6.8 \mathrm{~Hz}) 4.89$ - 5.08 (m, 4H) 5.73 - 5.93 (ddt, 2H, $\left.J_{l}=17.1, J_{2}=10.1, J_{3}=6.7 \mathrm{~Hz}\right) .{ }^{13} \mathrm{C}$ NMR $(75 \mathrm{MHz}$, CHLOROFORM- $d$ ) $\delta$ ppm 19.74, 27.11, 28.97, 29.19, 29.55, 29.66, 29.73, 30.05, 32.76, 33.85, 37.11, 114.05, 139.23. CG/EI-MS: [M] ${ }^{+}$ calcd for $\mathrm{C}_{24} \mathrm{H}_{46}, 334.3599$; found 334.3587. Elemental Analysis: calcd for $\mathrm{C}_{24} \mathrm{H}_{46}$ : C, 86.14; $\mathrm{H}$, 13.86. Found: C, 86.31; H, 13.85 .

\section{5 - Polymerization}

\section{ADMET polymerization: General procedure}

The monomer ( $\alpha, \omega$-diene) was added into a flame-dried Schlenk tube equipped with a stir bar. The liquid monomer was degassed by bubbling argon under stirring for 30 minutes. Vacuum was applied another 30 minutes to guarantee removal of dissolved oxygen and volatile contaminants. After that, the reaction flask was sealed and taken to a glove box where Schrock's catalyst was added (500:1 mol monomer to catalyst ratio). The reaction flask was then sealed under argon atmosphere and taken to a vacuum line. The polymerization proceeded at $70^{\circ} \mathrm{C}$ (oil bath) for 72 hours. After that, the highly viscous reaction mixture was dissolved in toluene and quenched by exposure to air under stirring at room temperature. The dissolved unsaturated polymer was precipitated into cold methanol and collected by vacuum filtration. Removal of residual toluene and methanol was carried out by drying the melted polymer under vacuum.

u-PE15-D 4 (5a) 


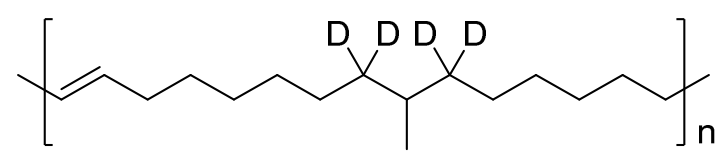

Hz) 1.26 (br, 17H) $1.81-2.21$ (br, 4H) $5.21-5.54(\mathrm{~m}, 2 \mathrm{H}) .{ }^{13} \mathrm{C}$ NMR $(75 \mathrm{MHz}$,

CHLOROFORM- $d$ ) $\delta$ ppm 19.63, 26.77, 26.89, 27.25, 29.24, 29.28, 29.41, 29.51, 29.63, 29.72, 29.88, 29.99, 32.41, 32.66, 129.86, 130.33. GPC data: $M_{n}=28$ 049; PDI $\left(M_{w} / M_{n}\right)=3.28$.

u-PE21-D 4 (5b)<smiles>[2H]C([2H])(CCCCCCCCC=C(C)C)C(C)C([2H])([2H])CCCCCCCC(C)(C)C</smiles>

Unsaturated polymer $\mathbf{5 b}$ was prepared from $1.12 \mathrm{~g}$ of $\mathbf{4 b}$. Obtained $953 \mathrm{mg}$ of solid material. $n{ }^{1} \mathrm{H}$

NMR $\quad(300 \quad \mathrm{MHz}$,

CHLOROFORM- $d$ ) $\delta$ ppm 0.84 (d, 3H, $J=6.4 \mathrm{~Hz}) 1.27$ (br, 29H) 1.98 (br, 4H) 5.24 - $5.49(\mathrm{~m}$, $2 \mathrm{H}) .{ }^{13} \mathrm{C}$ NMR (75 MHz, CHLOROFORM- $d$ ) $\delta$ ppm 19.85, 27.14, 27.44, 29.42, 29.56, 29.79, 29.91, 29.98, 30.23, 32.63, 32.85, 130.06, 130.53. GPC data: $M_{n}=46831 ; \operatorname{PDI}\left(M_{w} / M_{n}\right)=2.24$.

\section{u-PE15 (5c)}

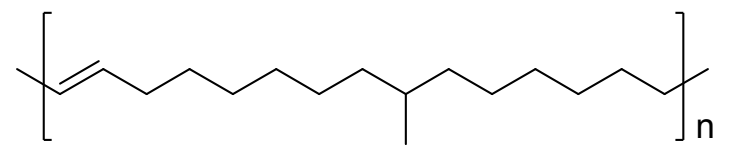

Unsaturated polymer $\mathbf{5 c}$ was prepared from $1 \mathrm{~g}$ of 4c. Obtained $840 \mathrm{mg}$ of soft material. ${ }^{1} \mathrm{H}$ NMR $(300$ MHz, CHLOROFORM- $d$ ) $\delta$ ppm $0.84(\mathrm{~d}, 3 \mathrm{H}, J=6.3$ $\mathrm{Hz}) 0.98-1.42(\mathrm{br}, 21 \mathrm{H}) 1.87-2.09$ (br, 4H) $5.27-5.49$ (m, 2H) ${ }^{13} \mathrm{C}$ NMR $(75 \mathrm{MHz}$, CHLOROFORM- $d$ ) $\delta$ ppm 19.73, 27.10, 29.27, 29.72, 29.92, 32.65, 32.78, 37.13, 130.33. GPC data: $M_{n}=68371 ; \operatorname{PDI}\left(M_{w} / M_{n}\right)=1.65$.

u-PE21 (5d)

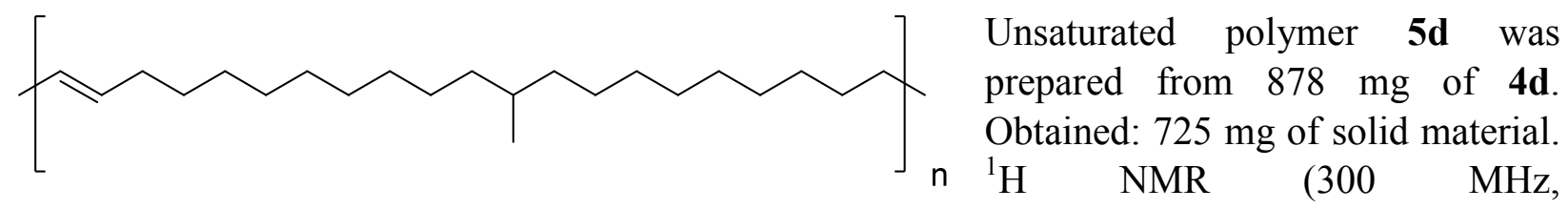

CHLOROFORM- $d$ ) $\delta$ ppm $0.84(\mathrm{~d}, 3 \mathrm{H}, J=6.3 \mathrm{~Hz}) 1.01$ - $1.44(\mathrm{br}, 33 \mathrm{H}) 1.97$ (br, 4H) $5.28-5.48$ $(\mathrm{m}, 2 \mathrm{H}) .{ }^{13} \mathrm{C}$ NMR $(75 \mathrm{MHz}$, CHLOROFORM- $d$ ) $\delta \mathrm{ppm} \mathrm{19.74,} \mathrm{27.13,} \mathrm{29.22,} \mathrm{29.58,} \mathrm{29.70,}$ $29.77,30.07,32.65,32.79,37.14,130.33$. GPC data: $M_{n}=29918$; PDI $\left(M_{w} / M_{n}\right)=2.32$.

\section{6-Saturated Polymers}

\section{General procedure for hydrogenation/deuteration}


Unsaturated polymer dissolved in toluene $(40 \mathrm{~mL})$ was added to a Parr bomb glass sleeve and degassed by bubbling argon for 30 minutes. Palladium on carbon (10 wt. \%) was added (15 $\mathrm{mg} / 100 \mathrm{mg}$ of unsaturated polymer) to the solution. The bomb was purged and charged with 800 psi of $\mathrm{H}_{2}(\mathbf{5 a - b}, \mathbf{6 a - b})$ or $\mathrm{D}_{2}$ gas $(\mathbf{5} \mathbf{c}-\mathbf{d}, \mathbf{6 c - d})$. The reaction was allowed to proceed for 72 hours at $80^{\circ} \mathrm{C}$ (oil bath) under stirring. The catalyst was removed by vacuum filtration through a Celite bed. The polymer solution was precipitated in cold methanol $(1 \mathrm{~L})$. The precipitate was filtered and dried under vacuum.

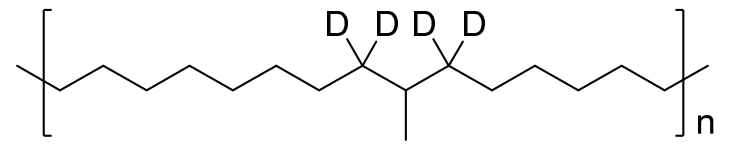

\section{PE15-D $_{4}(6 a)$}

CHLOROFORM- $d$ ) $\delta$ ppm 0.84 (d, 3H, $J=6.6 \mathrm{~Hz}) 1.16-1.36$ (br, 25H). ${ }^{13} \mathrm{C}$ NMR $(75 \mathrm{MHz}$, CHLOROFORM- $d$ ) $\delta$ ppm 19.81, 27.08, 29.90, 29.95, 30.19, 32.57. GPC data: $M_{n}=28$ 049; PDI $\left(M_{w} / M_{n}\right)=3.28$. DSC data: $\mathrm{Tm}=38.46^{\circ} \mathrm{C}, \Delta \mathrm{H}_{\mathrm{f}}=76.58 \mathrm{~J} / \mathrm{g} ; \mathrm{Tc}=27.53^{\circ} \mathrm{C}, \Delta \mathrm{H}_{\mathrm{c}}=78.77 \mathrm{~J} \mathrm{Jg}^{-1}$. TGA data: $\mathrm{T}_{95 \%}=396.29^{\circ} \mathrm{C}, \mathrm{T}_{50 \%}=427.92^{\circ} \mathrm{C}\left(\mathrm{T}_{\mathrm{x} \%}\right.$, temperature where $(100-\mathrm{x}) \%$ weight loss is observed).

\section{PE21-D $_{4}(6 b)$}

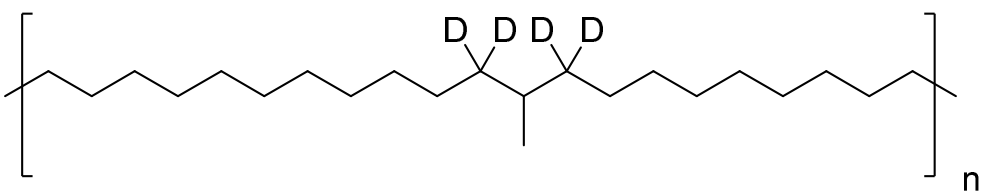

Polymer 6b was obtained from $0.900 \mathrm{~g}$ of $\mathbf{5 b}$. The saturated polymer $\mathbf{6 b}$ was obtained as an offn white solid. ${ }^{1} \mathrm{H}$ NMR $(300 \mathrm{MHz}$, CHLOROFORM- $d$ ) $\delta$ ppm $0.84(\mathrm{~d}, 3 \mathrm{H}, J=6.4 \mathrm{~Hz}) 1.13-1.40$ (br, 37H). ${ }^{13} \mathrm{C}$ NMR $(75 \mathrm{MHz}$, CHLOROFORM- $d$ ) $\delta$ ppm 19.65, 26.92, 29.74, 30.03, 32.40. GPC data: $M_{n}=60$ 091; PDI $\left(M_{w} / M_{n}\right)=2.24$. DSC data: $\mathrm{Tm}=62.73^{\circ} \mathrm{C}, \Delta \mathrm{H}_{\mathrm{f}}=103.3 \mathrm{~J} / \mathrm{g} ; \mathrm{Tc}=54.52^{\circ} \mathrm{C}, \Delta \mathrm{H}_{\mathrm{c}}=99.57 \mathrm{~J}^{-\mathrm{g}^{-1}}$. TGA data: $\mathrm{T}_{95 \%}=402.26^{\circ} \mathrm{C}, \mathrm{T}_{50 \%}=432.50^{\circ} \mathrm{C}$.

\section{PE15-D $_{2}(6 c)$}

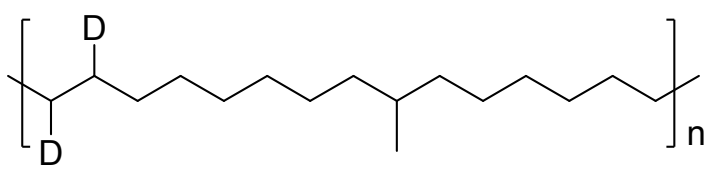

Polymer $\mathbf{6 c}$ was obtained from $0.426 \mathrm{~g}$ of $\mathbf{5 c}$. The saturated polymer $\mathbf{6 c}$ was obtained as an off-white solid $\quad(275 \quad \mathrm{mg}) . \quad{ }^{1} \mathrm{H} \quad \mathrm{NMR} \quad(300 \quad \mathrm{MHz}$, CHLOROFORM- $d$ ) $\delta$ ppm $0.84(\mathrm{~d}, 3 \mathrm{H}, J=6.3 \mathrm{~Hz})$ $0.98-1.46$ (br, $25 \mathrm{H}) .{ }^{13} \mathrm{C}$ NMR (75 MHz, CHLOROFORM- $d$ ) $\delta$ ppm 19.74, 27.12, 29.62, 29.72, 29.77, 30.07, 32.77, 32.12, 37.12. GPC data: $M_{n}=55$ 703; PDI $\left(M_{w} / M_{n}\right)=2.33$. DSC data:

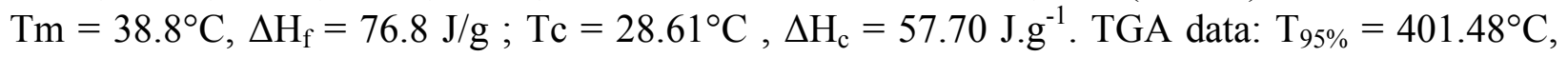
$\mathrm{T}_{50 \%}=429.27^{\circ} \mathrm{C}$. 


\section{PE21-D $_{2}$ (6d)}

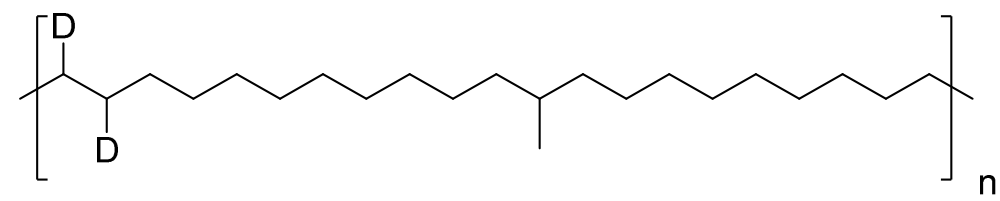

Polymer 6d was obtained from $0.370 \mathrm{~g}$ of $\mathbf{5 d}$. The saturated polymer 6a was obtained as an off(300 MHz, CHLOROFORM- $d$ ) $\delta$ ppm $0.84(\mathrm{~d}, 3 \mathrm{H}, J=6.3 \mathrm{~Hz}) 0.99-1.44(\mathrm{~m}, 37 \mathrm{H}) .{ }^{13} \mathrm{C}$ NMR (75 MHz, CHLOROFORM- $d$ ) $\delta$ ppm 19.76, 27.13, 29.62, 29.75, 30.07, 32.78, 37.13. GPC data: $M_{n}=27$ 628; PDI $\left(M_{w} / M_{n}\right)=2.16$. DSC data: $\mathrm{Tm}=61.7^{\circ} \mathrm{C}, \Delta \mathrm{H}_{\mathrm{f}}=100.8 \mathrm{~J}^{-1} \mathrm{~g}^{-1} ; \mathrm{Tc}=55.01^{\circ} \mathrm{C}$, $\Delta \mathrm{H}_{\mathrm{c}}=103.4 \mathrm{~J} / \mathrm{g}$. TGA data: $\mathrm{T}_{95 \%}=391.16^{\circ} \mathrm{C}, \mathrm{T}_{50 \%}=430.20^{\circ} \mathrm{C}$. 